\title{
58 THE COST-BENEFITS OF END-OF-LIFE TRAINING IN CARE HOMES WITH DEMENTIA PATIENTS
}

10.1136/bmjspcare-2011-000053.58

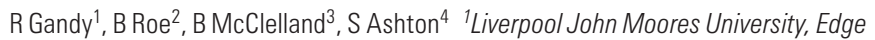
Hill University, UK; ${ }^{2}$ Personal Social Services Research Unit, University of Manchester, Manchester, UK; ${ }^{3}$ Centre for Public Sector Management, Liverpool Business School, Liverpool John Moores University, Liverpool, UK; ${ }^{4}$ Health and Applied Social Sciences, Liverpool John Moores University, Liverpool, UK

An education and training initiative took place in 2006 in Greater Manchester for staff in five local care homes.

Aims The aim was to improve the quality of end-of-life (EoL) care for older people with dementia, through the use of established EoL care tools, including advance care planning. As part of a full evaluation, an economic appraisal examined the activity, outcomes and finances.

Methods Data was collected on patients from the homes that died in the year before and the year after the initiative. The activity and costs of training and direct support to the homes were collected, with cost-benefit analyses subsequently undertaken.

Results The full evaluation showed many resultant quality benefits: improved communication between nursing, care and medical staff; better symptom management; and, support for family members and carers.

The proportion of patients that died in their preferred place of death increased from $48 \%$ to $63 \%$ following the initiative. Advance care plans were in place for $83 \%$ of those dying in the care homes, but none of those dying in hospital. The LCP was used for $67 \%$ of those dying in the care homes.

A cost-beneficial approach is affordable, but PCTs cannot expect cash savings, to fund education and training programmes, unless there are associated reductions in admissions to hospital, which is a primary aim of the Gold Standards Framework. ${ }^{2}$

Conclusions The analyses evidenced that training care home staff in EoL care tools is affordable and cost-beneficial, and quantified key variations relating to whether patients had advance care plans.

\section{REFERENCES}

1. Ashton, $\mathrm{S}$, et al. An end-of-life care initiative for people with dementia. Eur J Palliat Care, 2009;16(5), 240-3.

2. Gandy, R, et al. An economic appraisal of an end-of-life care training initiative for care homes with dementia patients. J Care Serv Manag, 2010;4(4):320-9. 